\title{
Nutritional Health Status of Rural Tribal Children in Khowai District of Tripura, India
}

\author{
Manabika Debbarma, Lord Litan Debbarma and Dipak Nath* \\ Krishi Vigyan Kendra, Divyodaya, Khowai, P.O. Chebri, Dist. Khowai, PIN 799207, \\ Tripura, India \\ *Corresponding author
}

\begin{tabular}{|l|}
\hline K e y w o r d s \\
$\begin{array}{l}\text { Nutritional health } \\
\text { status, Malnutrition, } \\
\text { Rural tribal children, } \\
\text { Khowai district }\end{array}$ \\
\hline Article Info \\
\hline $\begin{array}{l}\text { Accepted: } \\
\text { 20 August } 2018 \\
\text { Available Online: } \\
\text { 10 September } 2018\end{array}$ \\
\hline
\end{tabular}

A B S T R A C T

Under nutrition is a major public health problem among school children. Adequate nutrition is critical for optimal growth and health development of children. To assess the nutritional health of rural tribal 6-12 years school going children of Khowai district, Tripura. The sample for the present cross sectional study was conducted in three schools (Sukhiabari Jr. B School, R.S Colony Sr. B School and Kalidas Debbarma Smriti Sr. B School) rural based primary and Sr. B School of Khowai district, Tripura, in India. Total of 155 students (78 boys and 77 girls) of aged 6-12 years were participated in the study. The sample was selected by cluster random sampling method. The subjects consisted mostly from families of lower socio-economic class of Hindu and some were from Christian community and are consisted to be by typical of average Indian children. The children were assessed for nutritional status by clinical examination as well as anthropometric assessment. Weight and height of the children were measured and height-for-age (stunting), weight-for-height (wasting) and weight-for-age (under weight) were calculated. Out of 155 numbers of school children, wasting was found in $53.83 \%$ boys and $72.71 \%$ girls out of which $16.12 \%$ children showed severe degree of wasting. Stunting was found in $37 \%$ boys and $58.44 \%$. Rice consumption, family size, infection, vaccination, latrine availability were significantly associated with malnutrition. Hair changes were seen in $80.64 \%$. Teeth changes were seen in $56.12 \%$. Skin changes were noted in $65.80 \%$ children. The study provided evidence that school children in Sukhiabari village were under acute and chronic nutritional stress. Promoting appropriate dietary habits through effective nutritional education would be an effective preventive method. Main focus should be on qualitative and quantitative impartment on the diets with increased awareness on importance of preventing under-nutrition. Under-nutrition was pronounced in girls as compared to boys.

\section{Introduction}

According to 2011 census, the total population of India was 121 crores. Among them the total tribal population was $84,326,240$ and most of them belong to low Socio-economic status. The second smallest Sub-Himalayan hilly state named Tripura situated in the north-east region of India consists of Nine-teen classified tribal population. The total population of Tripura was 36, 71,032 (According to 2011 Census). Among them the total tribal population was 9, 93,426. Mostly they were situated in rural area. The rural tribal 
populations are recognized as socially and economically vulnerable. Khowai a district of Tripura situated in west of the Tripura map. The total population of Khowai was 3, 71,722 and the total tribal population was $1,65,532$ (According to 2011 Census). So many rural tribal villages are there in Khowai district. One of the villages in Sukhiabari ADC village, many poor families is living there. Their economic status and social status are very poor. In this reason there children's health status also will be poor.

Nutrition may be defined as the science of food and its relationship of health. It is concerned primarily with the part played by nutrients in body growth, development and maintenance. Good nutrition means "maintaining a nutritional status that enables us to grow well and enjoy good health".

Health is defined by the World Health Organization as a "state of complete physical, mental and social wellbeing (WHO, 1984). It is also asserted that health may be seen as a state of dynamic equilibrium between organism and its environmental. Good health corresponds to dynamic stability, normal function and homeostatic control. Ill-health corresponds to a state of instability, loss of function and failure of self-regulation. Globally, malnutrition among school age children is becoming a major public health concern. More than 200 million schools are stunted and underweight and if no action is taken and at this rate, about one billion school children will be growing up by 2020 with impaired physical and mental development (Srivastava et al., 2012). Malnutrition is the underlying cause of 7.6 million child deaths each year before their fifth birthday. Meeting this challenge is doubly urgent because among children who survive, chronic malnutrition causes devastating and irreversible damage (Chesire et al., 2008). Lack of nutritious food coupled with infection and illness, means their bodies and brains do not develop properly and at least 170 million children are affected by stunting (Global Monitoring Report, 2012; Garba et al., 2010; Reji et al., 2011). The term malnutrition refers to both under-nutrition and over nutrition. Good nutrition provides stronger immune system, better health and productivity. Various forms of malnutrition including both macro and micro nutrient, deficiencies affect a large segment of population in India (Vandana Sati et al., 2012). India has the dubious distinction of having the largest number of malnourished children in the world. Assessment of nutritional status is a vast subject (WHO, 1995, 1999). According to Joliffe, the study of nutritional status of any country is important for the mechanism of evaluation and morphological character which brings about the change in the pattern of body development and its structure. The social system of India are very surprising where the boys are always has been taken care well, the girls child by parents. So almost girl children are victim of negligence. Boys are given first priority with the available food within the family. The propose study provides an opportunity to investigate the nutritional health status of rural tribal 6-12 years boys and girls school going children. The possible effect of children health are malnutrition, under nutrition will be more pronounced in girls as compared to boys. The proposed study attempts to investigate this hypothesis.

\section{Materials and Methods}

\section{Study Samples}

The sample for the present cross sectional study was conducted in three schools (Sukhiabari Jr. B School, R.S Colony Sr. B School and Kalidas Debbarma Smriti Sr. B School) rural based primary and Sr. B School of Khowai district, Tripura, in India. Total of 155 students ( 78 boys and 77 girls) of aged 6- 
12 years were participated in the study. The sample was selected by cluster random sampling method. The subjects consisted mostly from families of lower socio-economic class of Hindu and some were from Christian community and are consisted to be by typical of average Indian children.

\section{Study area}

Tripura is a Sub- Himalayan hilly state of North -East India. According to the census report of 2011 the population of Tripura is about 36.71 lakhs with about 1.87 lakhs males and 1.79 lakhs females. Most of them belonged to low socio-economic status (Tripura: Human Development Report).

The sex ratio of the state is 961 females per thousand males. The density of the population is 350 persons per square kilometers. Tripura constitutes $0.3 \%$ of India's total population. They are scattered over 8 districts, 58 blocks and 870 villages.82.94 percent people of this state live in rural areas (Bhargava et al., 2006; Das, 2008; Uddin and Nag, 2012).

The total population of Khowai district was 3, 71,722 and the total tribal population was 1 , 65,532 (according to 2011 census). This study was conducted over three months i.e. August, 2015 and September, 2015. The selected schools are located approximately $16 \mathrm{~km}$ from khowai town.

\section{Data Collection}

Ethical approval was obtained from the Headmasters of the selected schools and guardians of the chosen families respectively before commencement of the study. All children between 6-12 years of age as determined using schools records were included in the study. Study aim, plan and benefits were explained to each school H/M to obtain his/her approval.

\section{Anthropometric measurement}

The measurements were performance by following the standard techniques (Laurie and Wiener, 1981). Height, Weight, Body Mass Index (BMI), Mid-upper-arm Circumference (MUAC) was taken in to consideration. Height in centimeter was marked on a wall in the school with the help of a measuring tape. All children were measured against the wall. Height was recorded to the nearest $1 \mathrm{~cm}$ (Anand et al., 1999). Body weight was measured with light clothing and shoes off, buy weighting machine to the nearest $200 \mathrm{~g}$. Mid-upper-arm-circumference (MUAC) was measured in at the level of the midpoint of the upper arm (left arm), between the acroming process and the tip of the olecranon, with the arm hanging relaxed by an accurate soft metallic tape (Stanley made in England0 to the nearest $0.1 \mathrm{~cm}$ range (Gopaldas et al., 1987).

To assess the nutritional status of an individual or population, anthropometry is widely recognized as one of the useful techniques because it is highly sensitive to detect under-nutrition (National Institute of Nutrition, 2005). Gomez classification and water low's classification were applied to calculate the stunting (Height for age) and underweight (weight-for age) types of malnutrition to explain the level of their physical growth and development (Gomez et al., 1956).

\section{Questionnaire Survey}

First, school children were screened at school to participate in the study and then parents were traced back for detail information (Interview) on personal (Student), and parental socio-economic, demographic, environmental and access to facilities and health and health related issues at the community level. Data on the type of most frequently produced add consumed crop, households assets and other 
aspects were collected during interview by students' parents/ family members.

\section{Clinical nutrition survey}

Clinical examination is commonly used in survey, since it is relatively simple and do not call for Sophisticated equipment. It revealed the anatomical changes due to malnutrition that can be diagnosed by the naked eyes.

\section{Food frequency questionnaire}

Food Frequency Questionnaire is either interviewer administrated or self-completed. A detailed questionnaire includes the list of foods and the subject answer as to how often and in what quantity each food is eaten per day, per weeks. The collected information of the food consumed is taken checked with the recommended Dietary Allowance (RDA by ICMR, 1990). It gives an estimate of the amount and frequency of the various nutrients consumed by the individual.

\section{Statistical analysis of data}

All the data were evaluated statistically by two tail's test was performed using the origin Lab (Ver.6.0) Software. P values of less than 0.05 were considered to indicate statistical significance.

\section{Results and Discussion}

Demographic characteristics, Housing and environmental health factors, health and health related factors, nutritional deficiencies, different nutritional anthropometric indices and nutrients consumption were examined to determine the nutritional health status of rural tribal children in the age group of 6-12 years school going children.

School age is considered as a dynamic period of growth and development because children undergo physical, mental, emotional and social changes. In other words the foundation of good health and sound mind are laid during the school age period (Srivastava et al., 2012). Hence the present study was formulated with the objective, to determine the Nutritional Health Status of rural tribal children.

A total of 155 numbers of school children from three Primaries and Sr. Basic Schools took part in the study. Mothers with Madhyamik pass were only $1.29 \%$ and $3.22 \%$ for fathers. Hindu was the most frequently appeared parental religion $92.25 \%$ and only $7.74 \%$ was Christian. Ownership of livestock was $95.48 \%$ and most type of crop they were used in rice (100\%). The percentage of latrine availability was $34.19 \%$ and refuse disposal was found $100 \%$ in open field. Animal living in the same house was $12.25 \%$. The source of drinking water for $17.41 \%$ of house hold was found to be pipe water, $8.38 \%$ was found protected well. Student's presence of dirt in finger nails was found $95.48 \%$ and wearing of shoes was found $62.58 \%$. The total percentage distribution of the nutritional deficiency signs amongst rural tribal children was found only $1.28 \%$ boys and $3.89 \%$ girls heaving water eyes. Lips were found $27.26 \%$ girls and $11.53 \%$ boys were children were suffering mild angular stomatitis. Only $29.48 \%$ of boys and only $9.09 \%$ of girls observed normal Hair. Teeth were observed $35.89 \%$ boys and $68.83 \%$ girls had discolored teeth which could be a result of poor dental care.

Physical examination of all the 155 children was carried out $78(50.32 \%)$ were boys and 77 $(49.67 \%)$ were girls. In present study boys were found taller than the girls. Saheb and Rajendra Prasad, (2009) in his study similarly found that which showed the boys being slightly taller than girls up to the age of 9 years. Both boys and girls height increases monotonically with their age (Table 1-14). 

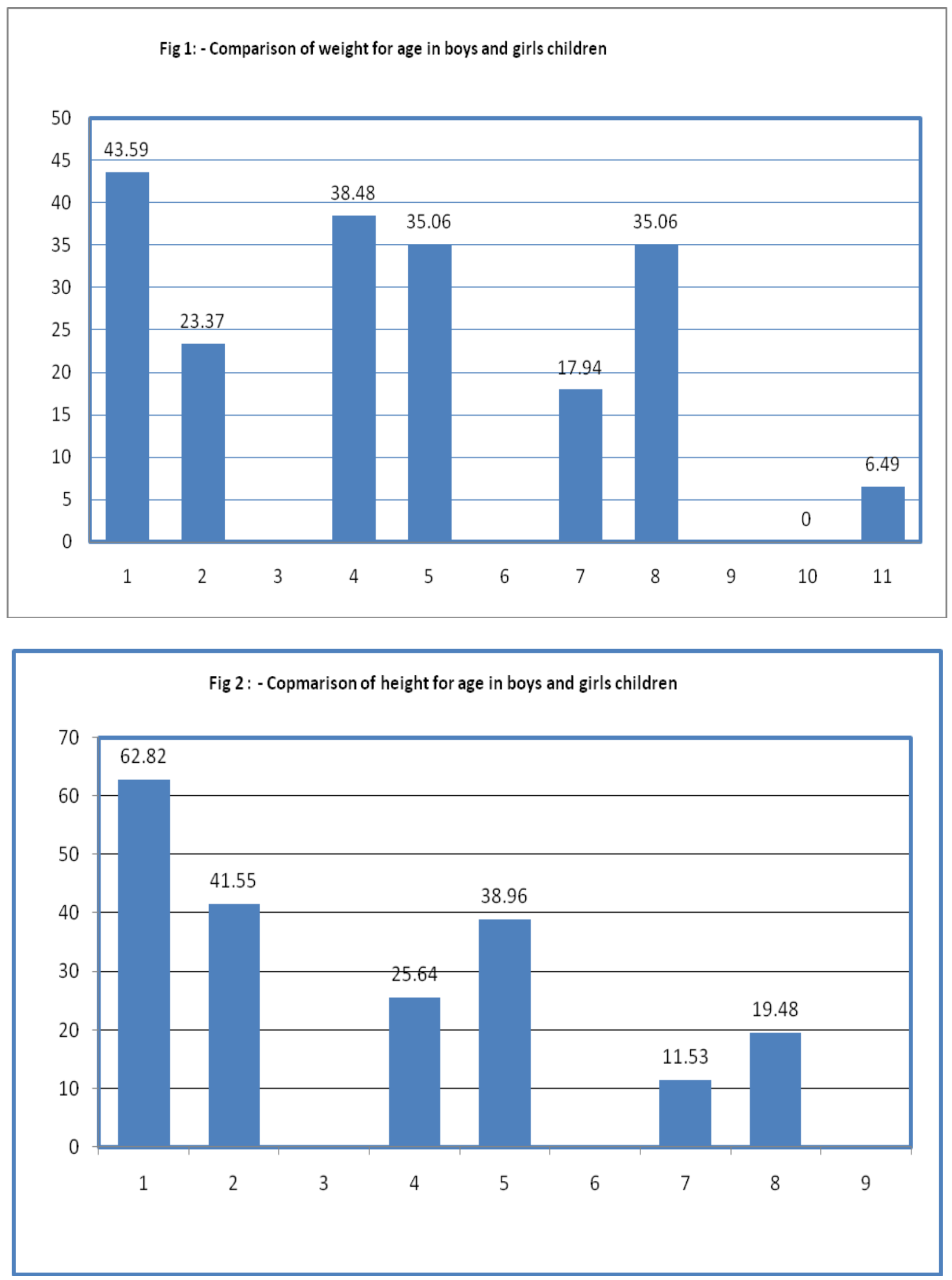
Table.1 Socio-economic and demographic characteristics of parents

\begin{tabular}{|c|c|c|}
\hline Variable & Category & n $(\%)$ \\
\hline Age of Mother & $\begin{array}{l}22-35 \\
36-45 \\
>45\end{array}$ & $\begin{array}{l}106(68.38 \%) \\
47(30.32 \%) \\
2(1.29 \%)\end{array}$ \\
\hline Age of Father & $\begin{array}{l}24-35 \\
36-45 \\
>45\end{array}$ & $\begin{array}{l}67(43.22 \%) \\
53(34.19 \%) \\
35(22.58 \%)\end{array}$ \\
\hline Marital status of Mothers & $\begin{array}{l}\text { Married } \\
\text { Divorced } \\
\text { Widowed }\end{array}$ & $\begin{array}{l}146(94.19 \%) \\
2(1.29 \%) \\
7(4.51 \%)\end{array}$ \\
\hline Age of School Children & $\begin{array}{l}6-9 \\
10-12\end{array}$ & $\begin{array}{l}89(57.41 \%) \\
66(42.58 \%)\end{array}$ \\
\hline Sex of School Children & $\begin{array}{l}\text { Male } \\
\text { Female }\end{array}$ & $\begin{array}{l}78(50.32 \%) \\
77(49.67 \%)\end{array}$ \\
\hline Religions of Parents & $\begin{array}{l}\text { Hindu } \\
\text { Muslim } \\
\text { Christian } \\
\text { Others }\end{array}$ & $\begin{array}{l}143(92.25 \%) \\
- \\
12(7.74 \%) \\
-\end{array}$ \\
\hline Mothers Education & $\begin{array}{l}\text { Does not read/ Write } \\
\text { Primary education } \\
\text { Madhyamik Pass }\end{array}$ & $\begin{array}{l}7(4.51 \%) \\
146(94.19 \%) \\
2(1.92 \%)\end{array}$ \\
\hline Father Education & $\begin{array}{l}\text { Does not read/ Write } \\
\text { Primary education } \\
\text { Madhyamik Pass }\end{array}$ & $\begin{array}{l}6(3.87 \%) \\
144(92.90 \%) \\
5(3.22 \%)\end{array}$ \\
\hline Occupation of Father & $\begin{array}{l}\text { Farmer } \\
\text { Daily Labours Government } \\
\text { Employee }\end{array}$ & $\begin{array}{l}103(66.45 \%) \\
47(30.32 \%) \\
5(3.22 \%)\end{array}$ \\
\hline Family Income & $\begin{array}{l}2000-3000 \\
3000-5000 \\
>5000\end{array}$ & $\begin{array}{l}43(27.74 \%) \\
107(69.03 \%) \\
5(3.22 \%)\end{array}$ \\
\hline Family size & $\begin{array}{l}2-5 \\
6-8 \\
>8\end{array}$ & $\begin{array}{l}71(45.80 \%) \\
81(52.25 \%) \\
3(1.93 \%)\end{array}$ \\
\hline Ownership of Radio & $\begin{array}{l}\text { Yes } \\
\text { No }\end{array}$ & $\begin{array}{l}- \\
155(100 \%)\end{array}$ \\
\hline Ownership of T.V & $\begin{array}{l}\text { Yes } \\
\text { No }\end{array}$ & $\begin{array}{l}87(56.12 \%) \\
68(43.87 \%)\end{array}$ \\
\hline Family decision maker & $\begin{array}{l}\text { Father } \\
\text { Mother } \\
\text { Both by discussion }\end{array}$ & $\begin{array}{l}93(60 \%) \\
49(31.61 \%) \\
13(23.63 \%)\end{array}$ \\
\hline Ownership of livestock & $\begin{array}{l}\text { Yes } \\
\text { No }\end{array}$ & $\begin{array}{l}148(95.48 \%) \\
7(4.51 \%)\end{array}$ \\
\hline Most type of crop & $\begin{array}{l}\text { Rice } \\
\text { Others }\end{array}$ & $\begin{array}{l}155(100 \%) \\
-\end{array}$ \\
\hline
\end{tabular}


Table.2 Housing and environmental health factors of parents of school children's

\begin{tabular}{|c|c|c|}
\hline Variable & Category & n $(\%)$ \\
\hline Type of roof material & $\begin{array}{l}\text { Thatched } \\
\text { Corrugated Iron }\end{array}$ & $\begin{array}{l}3(1.93 \%) \\
152(98.06 \%)\end{array}$ \\
\hline $\begin{array}{l}\text { Animal living in the same } \\
\text { house }\end{array}$ & $\begin{array}{l}\text { Yes } \\
\text { No }\end{array}$ & $\begin{array}{l}19(12.25 \%) \\
136(87.74 \%)\end{array}$ \\
\hline $\begin{array}{l}\text { Number of rooms of the } \\
\text { house }\end{array}$ & $\begin{array}{l}1 \\
2 \\
3 \\
4 \\
5\end{array}$ & $\begin{array}{l}39(25.16 \%) \\
100(64.51 \%) \\
7(4.51 \%) \\
9(5.80 \%) \\
-\end{array}$ \\
\hline Latrine availability & $\begin{array}{l}\text { Yes } \\
\text { No }\end{array}$ & $\begin{array}{l}53(34.19 \%) \\
102(65.80 \%)\end{array}$ \\
\hline Refuse disposal & $\begin{array}{l}\text { Pit } \\
\text { Burning } \\
\text { Open field } \\
\text { Garbage can }\end{array}$ & $\begin{array}{l}- \\
- \\
155(100 \%)\end{array}$ \\
\hline Source of drinking water & $\begin{array}{l}\text { Pipe water } \\
\text { Protected Well/ Spring } \\
\text { Unprotected Well/Spring } \\
\text { River }\end{array}$ & $\begin{array}{l}27(17.41 \%) \\
13(8.38 \%) \\
115(74.19 \%)\end{array}$ \\
\hline
\end{tabular}

Table.3 Health and health related factors of School Children

\begin{tabular}{|c|c|c|}
\hline Variable & Category & n $(\%)$ \\
\hline $\begin{array}{l}\text { Infection during previous } \\
\text { two weeks }\end{array}$ & $\begin{array}{l}\text { Yes } \\
\text { No }\end{array}$ & $\begin{array}{l}15(9.67 \%) \\
140(90.32 \%)\end{array}$ \\
\hline $\begin{array}{l}\text { Knowledge of mothers on } \\
\text { good for children }\end{array}$ & $\begin{array}{l}\text { Yes } \\
\text { No }\end{array}$ & $\begin{array}{l}87(56.12 \%) \\
68(43.87 \%)\end{array}$ \\
\hline Provision pocket money & $\begin{array}{l}\text { Yes } \\
\text { No }\end{array}$ & - $155(100 \%)$ \\
\hline Immunization status & $\begin{array}{l}\text { Yes } \\
\text { No }\end{array}$ & $\begin{array}{l}148(95.48 \%) \\
7(4.51 \%)\end{array}$ \\
\hline $\begin{array}{l}\text { Number of breast milk } \\
\text { feeding years }\end{array}$ & $\begin{array}{l}\text { Up to six months } \\
\text { One year } \\
\text { Two years and above }\end{array}$ & $\begin{array}{l}27(17.41 \%) \\
116(74.83 \%) \\
12(7.74 \%)\end{array}$ \\
\hline $\begin{array}{l}\text { Presence of dirt in finger } \\
\text { nails }\end{array}$ & $\begin{array}{l}\text { Yes } \\
\text { No }\end{array}$ & $\begin{array}{l}148(95.48 \%) \\
7(4.51 \%)\end{array}$ \\
\hline Wearing of shoes & $\begin{array}{l}\text { Yes } \\
\text { No }\end{array}$ & $\begin{array}{l}97(62.58 \%) \\
58(37.41 \%)\end{array}$ \\
\hline
\end{tabular}


Table.4 Clinical nutritional survey chart of rural tribal children's

\begin{tabular}{|c|c|c|c|}
\hline Clinical signs & Category & Boys & Girls \\
\hline General appearance & $\begin{array}{l}\text { Good } \\
\text { Fair } \\
\text { Poor } \\
\text { Very poor }\end{array}$ & $\begin{array}{l}33(42.30 \%) \\
28(35.89 \%) \\
16(20.51 \%) \\
1(1.28 \%)\end{array}$ & $\begin{array}{l}18(23.37 \%) \\
28(35.06 \%) \\
25(32.46 \%) \\
7(9.09 \%)\end{array}$ \\
\hline Hair & $\begin{array}{l}\text { Normal } \\
\text { Loss of Luster } \\
\text { Discolored and Dry } \\
\text { Sparse and Brittle }\end{array}$ & $\begin{array}{l}23(29.48 \%) \\
17 .(21.79 \%) \\
31(39.74 \%) \\
7(8.97 \%)\end{array}$ & $\begin{array}{l}7(9.09 \%) \\
20(25.97 \%) \\
42(54.54 \%) \\
8(10.38 \%)\end{array}$ \\
\hline Eye discharge & $\begin{array}{l}\text { Absent } \\
\text { Watery } \\
\text { Mucopurulent }\end{array}$ & $\begin{array}{l}77(98.71 \%) \\
1(1.28 \%)\end{array}$ & $\begin{array}{l}74(96.10 \%) \\
3(3.89 \%)\end{array}$ \\
\hline Lips & $\begin{array}{l}\text { Normal Angular } \\
\text { Stomatitis, } \\
\text { Mild } \\
\text { Stomatistis, } \\
\text { Marked }\end{array}$ & $\begin{array}{l}67(85.89 \%) \\
9(11.53 \%) \\
2(2.56 \%)\end{array}$ & $\begin{array}{l}56(72.72 \%) \\
11(14.28 \%) \\
10(12.98 \%)\end{array}$ \\
\hline Teeth & $\begin{array}{l}\text { Absent } \\
\text { Chalky teeth } \\
\text { Pitting of teeth } \\
\text { Discolored }\end{array}$ & $\begin{array}{l}47(60.25 \%) \\
2(2.56 \%) \\
1(1.28 \%) \\
28(35.89 \%)\end{array}$ & $\begin{array}{l}21(27.27 \%) \\
3(3.89 \%) \\
- \\
53(68.83 \%)\end{array}$ \\
\hline Skin & $\begin{array}{l}\text { Normal } \\
\text { Loss of Luster } \\
\text { Dry and Rough }\end{array}$ & $\begin{array}{l}31(39.74 \%) \\
12(15.38 \%) \\
35(44.87 \%)\end{array}$ & $\begin{array}{l}22(28.57 \%) \\
11(14.28 \%) \\
44(57.14 \%)\end{array}$ \\
\hline
\end{tabular}

Table.5 Distribution of boy's and girl's according to their age

\begin{tabular}{|l|l|l|l|l|l|}
\hline $\begin{array}{l}\text { Age } \\
\text { years) }\end{array}$ & $\begin{array}{l}\text { complete } \\
\text { Number } \\
\text { Children }\end{array}$ & of & Boy's & \multicolumn{2}{l|}{ Girl's } \\
\cline { 4 - 6 } & No & $\%$ & No & $\%$ \\
\hline 6 & $22(14.19)$ & 12 & 54.54 & 10 & 45.45 \\
\hline 7 & $23(14.83)$ & 11 & 47.82 & 12 & 52.17 \\
\hline 8 & $22(14.19)$ & 11 & 50 & 11 & 50 \\
\hline 9 & $22(14.19)$ & 11 & 50 & 11 & 50 \\
\hline 10 & $23(14.83)$ & 11 & 47.82 & 12 & 52.61 \\
\hline 11 & $21(13.54)$ & 11 & 52.38 & 10 & 47.61 \\
\hline 12 & $22(14.19)$ & 11 & 50 & 11 & 50 \\
\hline Total (\%) & $155(100)$ & 78 & 50.32 & 77 & 49.67 \\
\hline
\end{tabular}


Table.6 Mean height of the boys and girls

\begin{tabular}{|c|c|c|c|c|c|c|c|c|}
\hline \multirow[t]{2}{*}{ Age } & \multicolumn{3}{|c|}{ Boy's } & \multicolumn{3}{|c|}{ Girl's } & \multirow{2}{*}{$\begin{array}{l}\text { t } \\
\text { value }\end{array}$} & \multirow{2}{*}{$\begin{array}{l}\mathbf{p} \\
\text { value }\end{array}$} \\
\hline & No & $\begin{array}{l}\text { Mean } \\
\text { height } \\
\text { (cm) }\end{array}$ & SD & No & $\begin{array}{l}\text { Mean } \\
\text { height } \\
\text { (cm) }\end{array}$ & SD & & \\
\hline 6 & 12 & 114.33 & 3.47 & 10 & 111.7 & 2.36 & 0.058 & $\mathrm{P}<0.008$ \\
\hline 7 & 11 & 119.90 & 4.71 & 12 & 118.33 & 5.51 & 0.751 & $\mathrm{P}<0.008$ \\
\hline$\overline{8}$ & 11 & 123.90 & 4.39 & 11 & 120.54 & 3.60 & 0.080 & $\mathrm{P}<0.008$ \\
\hline 9 & 11 & 134 & 6.94 & 11 & 124 & 3.64 & 0.0006 & $\mathrm{P}<0.007$ \\
\hline 10 & 11 & 131.63 & 7.28 & 12 & 130.75 & 6.84 & 0.777 & $\mathrm{P}<0.007$ \\
\hline 11 & 11 & 133 & 6.61 & 10 & 136.5 & 8.60 & 0.338 & $\mathrm{P}<0.007$ \\
\hline 12 & 11 & 146 & 5.32 & 11 & 140.27 & 6.41 & 0.577 & $\mathrm{P}<0.006$ \\
\hline
\end{tabular}

Table.7 Mean weight of the boys and girls

\begin{tabular}{|c|c|c|c|c|c|c|c|c|}
\hline \multirow[t]{2}{*}{ Age } & \multicolumn{3}{|c|}{ Boy's } & \multicolumn{3}{|c|}{ Girl's } & \multirow{2}{*}{$\begin{array}{l}\mathrm{t} \\
\text { value }\end{array}$} & \multirow{2}{*}{$\begin{array}{l}\mathbf{p} \\
\text { value }\end{array}$} \\
\hline & No & $\begin{array}{l}\text { Mean } \\
\text { weight } \\
\text { (kg) }\end{array}$ & SD & No & $\begin{array}{l}\text { Mean } \\
\text { weight } \\
\text { (kg) }\end{array}$ & SD & & \\
\hline 6 & 12 & 18.16 & 1.40 & 10 & 16.3 & 2.1 & 0.037 & $\mathrm{P}<0.05$ \\
\hline 7 & 11 & 22.54 & 3.36 & 12 & 20.33 & 4.74 & 0.0229 & $\mathrm{P}<0.04$ \\
\hline 8 & 11 & 21.81 & 4.01 & 11 & 19.27 & 2.83 & 0.117 & $P<0.04$ \\
\hline 9 & 11 & 23.63 & 4.53 & 11 & 19.63 & 2.49 & 0.025 & $\mathrm{P}<0.04$ \\
\hline 10 & 11 & 28 & 3.64 & 12 & 24.33 & 4.30 & 0.048 & $\mathrm{P}<0.03$ \\
\hline 11 & 11 & 34.36 & 5.84 & 10 & 32.4 & 5 & 0.440 & $\mathrm{P}<0.02$ \\
\hline 12 & 11 & 32.72 & 5.04 & 11 & 31 & 4.15 & 0.413 & $\mathrm{P}<0.03$ \\
\hline
\end{tabular}

Table.8 Nutritional status (weight for height) as per age group

\begin{tabular}{|l|l|l|l|l|l|l|l|l|l|}
\hline Age & No & Normal & $\%$ & \multicolumn{2}{|l|}{ Malnutrition } & & & \\
& & & & Mild & $\%$ & Moderate & $\%$ & Severe & $\%$ \\
\hline 6 & 22 & 8 & 36.36 & 8 & 36.36 & 5 & 22.72 & 1 & 4.54 \\
\hline $\mathbf{7}$ & 23 & 18 & 78.26 & 5 & 21.73 & - & - & - & - \\
\hline $\mathbf{8}$ & 22 & 7 & 31.81 & 4 & 18.18 & 6 & 27.27 & 5 & 22.72 \\
\hline $\mathbf{9}$ & 22 & 2 & 9.09 & 5 & 22.72 & 7 & 31.81 & 8 & 36.36 \\
\hline 10 & 23 & 7 & 30.43 & 4 & 17.39 & 8 & 34.78 & 4 & 17.39 \\
\hline 11 & 21 & 11 & 52.38 & 5 & 23.80 & 3 & 14.28 & 2 & 9.52 \\
\hline 12 & 22 & 4 & 18.18 & 4 & 18.18 & 9 & 40.90 & 5 & 22.72 \\
\hline
\end{tabular}


Int.J.Curr.Microbiol.App.Sci (2018) 7(9): 3170-3183

Table.9 Nutritional status (weight for height) as per sex

\begin{tabular}{|c|c|c|c|c|c|c|c|c|c|}
\hline \multirow[t]{3}{*}{ Sex } & \multirow[t]{3}{*}{ No } & \multicolumn{8}{|c|}{ Nutritional status } \\
\hline & & \multirow[t]{2}{*}{ Normal } & \multirow{2}{*}{$\begin{array}{l}\text { No } \\
(\%)\end{array}$} & \multicolumn{6}{|c|}{ Wasting } \\
\hline & & & & Mild & $\%$ & Moderate & $\%$ & Severe & $\%$ \\
\hline Boy's & 78 & 36 & 46.15 & 18 & 23.07 & 18 & 23.07 & 6 & 7.69 \\
\hline Girl's & 77 & 21 & 27.27 & 17 & 22.07 & 20 & 25.97 & 19 & 24.67 \\
\hline
\end{tabular}

Table.10 Nutritional status (Height for age) as per age

\begin{tabular}{|c|c|c|c|c|c|c|c|c|c|}
\hline \multirow[t]{3}{*}{ Age } & \multirow[t]{3}{*}{ No } & \multicolumn{8}{|c|}{ Nutritional status } \\
\hline & & \multirow[t]{2}{*}{ Normal } & \multirow[t]{2}{*}{$\%$} & \multicolumn{6}{|c|}{ Stunting } \\
\hline & & & & Mild & $\%$ & Moderate & $\%$ & Severe & $\%$ \\
\hline 6 & 22 & 15 & 68.18 & 7 & 31.81 & - & - & - & - \\
\hline 7 & 23 & 16 & 69.56 & 7 & 30.43 & - & - & - & - \\
\hline 8 & 22 & 13 & 59.09 & 8 & 36.36 & 1 & 4.54 & - & - \\
\hline 9 & 22 & 13 & 59.09 & 6 & 27.27 & 3 & 13.63 & - & - \\
\hline 10 & 23 & 9 & 39.13 & 7 & 30.43 & 7 & 30.43 & - & - \\
\hline 11 & 21 & 6 & 28.57 & 5 & 23.80 & 10 & 47.61 & - & - \\
\hline 12 & 22 & 9 & 40.90 & 9 & 40.90 & 4 & 18.18 & - & - \\
\hline
\end{tabular}

Table.11 Nutritional status (Height for age) as per sex

\begin{tabular}{|c|c|c|c|c|c|c|c|c|c|}
\hline \multirow[t]{2}{*}{ Sex } & \multirow[t]{2}{*}{ No } & \multirow[t]{2}{*}{ Normal } & \multirow[t]{2}{*}{$\%$} & \multicolumn{6}{|c|}{ Stunting } \\
\hline & & & & Mild & $\%$ & Moderate & $\%$ & Severe & $\%$ \\
\hline Boy's & 78 & 49 & 62.82 & 20 & 25.64 & 9 & 11.53 & - & - \\
\hline Girl's & 77 & 32 & 41.55 & 30 & 38.96 & 15 & 19.48 & - & - \\
\hline
\end{tabular}

Table.12 Nutritional status (Weight for age) as per age

\begin{tabular}{|c|c|c|c|c|c|c|c|c|c|}
\hline \multirow[t]{2}{*}{ Age } & \multirow[t]{2}{*}{ No } & \multirow[t]{2}{*}{ Normal } & \multirow[t]{2}{*}{$\%$} & \multicolumn{6}{|c|}{ Under nutrition } \\
\hline & & & & Mild & $\%$ & Moderate & $\%$ & Severe & $\%$ \\
\hline$\overline{6}$ & 22 & 8 & 36.36 & 11 & 50 & 3 & 13.63 & - & - \\
\hline 7 & 23 & 13 & 56.52 & 8 & 34.78 & 2 & 8.69 & - & - \\
\hline 8 & 22 & 7 & 31.81 & 6 & 27.27 & 9 & 40.90 & - & - \\
\hline 9 & 22 & 2 & 9.09 & 7 & 31.81 & 10 & 45.45 & 3 & 13.69 \\
\hline 10 & 23 & 7 & 30.43 & 10 & 43.47 & 4 & 17.39 & 2 & 8.69 \\
\hline 11 & 21 & 11 & 52.38 & 8 & 38.09 & 2 & 9.52 & - & - \\
\hline 12 & 22 & 4 & 18.18 & 7 & 31.81 & 11 & 50 & - & - \\
\hline
\end{tabular}


Table.13 Nutritional status (Weight for age) as per sex

\begin{tabular}{|c|c|c|c|c|c|c|c|c|c|}
\hline \multirow[t]{3}{*}{ Sex } & \multirow[t]{3}{*}{ No } & \multicolumn{8}{|c|}{ Nutritional status } \\
\hline & & \multirow{2}{*}{ Normal } & \multirow[t]{2}{*}{$(\%)$} & \multicolumn{6}{|c|}{ Under nutrition } \\
\hline & & & & Mild & $\%$ & Moderate & $\%$ & Severe & $\%$ \\
\hline Boy's & 78 & 34 & 43.59 & 30 & 38.46 & 14 & 17.94 & - & - \\
\hline Girl's & 77 & 18 & 23.37 & 27 & 35.06 & 27 & 35.06 & 5 & 6.49 \\
\hline
\end{tabular}

Table.14 Percentage of distribution of rural tribal children based on 'Frequency of Food Groups' intake

\begin{tabular}{|l|l|l|l|l|l|}
\hline $\begin{array}{l}\text { Sl. } \\
\text { No }\end{array}$ & Food Groups & Daily & Weekly & $\begin{array}{l}\text { Twice } \\
\text { month }\end{array}$ & a \\
\hline $\mathbf{1}$ & Cereals & $100 \%$ & - & - & - \\
\hline 2 & Pulses and Legumes & $122 \%$ & $22 \%$ & $57 \%$ & $9 \%$ \\
\hline 3 & Milk and Milk products & $41 \%$ & $7 \%$ & - & - \\
\hline 4 & $\begin{array}{l}\text { Green leafy vegetables } \\
\text { Fats and Oils }\end{array}$ & $71 \%$ & $29 \%$ & - & - \\
\hline 5 & $\begin{array}{l}\text { Meat, Egg, Fish and } \\
\text { Poultry }\end{array}$ & $17 \%$ & $78 \%$ & $17 \%$ & - \\
\hline 6 & & $5 \%$ & - \\
\hline
\end{tabular}

The peak increase in height boys was between 11 and 12 years and in girls it was 6 and 7 years. The study by Kalil and Khan (2004) show that overall increase in a mean height is more in boys $(40.52 \mathrm{~cm})$ than girls $(37.35 \mathrm{~cm})$ which similar in present study boys $(146 \mathrm{~cm})$ and girls $140.27 \mathrm{~cm}$ ). Maximum increase in mean weight has been seen 11 years of age in both boys and girls children. The study by Saheb and Rajendra Prasad, (2009) showed that the mean weight for boys and girls 6 years is $18.26 \mathrm{~kg}$ and $17.72 \mathrm{~kg}$ respectively. This study is similar and shows that the mean weight for boys and girls at 6 years is 18.16 $\mathrm{kg}$ and $16.3 \mathrm{~kg}$ respectively. The peak increase in weight was seen between 10 and 11 years in both boys and girls children.

Nutritional status according to water lows classification "weight for height" normal, mild, moderate and sever wasting in 6 year was found $36.36 \%, 36.36 \%, 22.72 \%$ and $4.54 \%$ respectively. Wasted children were more in girls than the boys. Kalil and Khan
(2004) present study is slightly different from the study which showed the age group of 10 to 12 was observed to be at the highest $(31.81 \%)$ risk of wasting. The sex was distribution of weight for height found only $46.15 \%$ boys and $27.27 \%$ girls was normal and $53.85 \%$ boys and $72.73 \%$ girls were suffering from different degrees of malnutrition. The nutritional status according to water lows classification (height for age) was found children of 11 ages were having very high percentage $(47.61 \%)$ of moderately impaired nutritional status. (Panda et al., 2000 ) is reported School children of Ludhiana city reported mild stunting in only $20.7 \%$ children were moderate and sever degree of stunting $5.5 \%$ and $5.6 \%$ children respectively.

According to sex wise distribution it showed that boys were more normal than the girls. Similarly the number and percentages of girls with mild and moderately impaired nutritional status were higher than the boys. According to weight for age maximum number of 
moderate malnutrition was found 8,9 and 12 years of age group, which is very much alarming. A study conducted by Sundaram et $a l$., in school children of Madras city reported prevalence of grade I malnutrition as $30.5 \%$ of grade II malnutrition as $42.5 \%$ and of grade III malnutrition as $21.5 \%$ (Sundaram et al., 1978). The percentage of severe malnutrition was seen in the age group of 9 and 10. The sex wise distribution of nutritional status and found $55.86 \%$ boys and $76.61 \%$ girls were suffering from different degrees of malnutrition. In sex wise distribution it showed that boys were more normal, mild, and moderate than the girls. In compare to boys and girls nutritional status it observed boys were more normal than the girl's children. Regarding the possible explanations of such results, it may be started that the illiteracy of mother, poverty and children frequently suffer from different type of diseases.

In the present study total of 155 students ( 78 boys and 77 girls) of aged group 6-12 years rural tribal children were participate. The study found that the nutritional status for both boys and girls were very poor. According to water low's classification, 53.85\%boys and $72.73 \%$ girls and $37.17 \%$ boys and $58.44 \%$ girls were suffering from different degrees of wasting and stunting gradually. It's noticed that the nutritional status as per 'weight for height' focused more severity than the nutritional status as per 'height for age'. According to Gomez classification, $56.4 \%$ bots and $70.66 \%$ girls were suffering from different degrees of under nutrition. It comparison to height, the weight of the children was showing less severity. The major factors identified for this problem is illiteracy of mother, because literacy and schooling levels, especially of mothers, are known to affect the style and goals of child care. In present study $62.58 \%$ mother were illiterate, this could have been a factor of poor health conditions of children. Other caused of poor nutritional status of children may be poor family background of the children, lack of well latrine facility and unprotected source of drinking water.

In the study, compare with boys and girls nutritional health status. It's appeared that wasting, stunting and under nutrition was much higher in girls $72.71 \%, 58.44 \%$ and $76.61 \%$ respectively than the boys $53.83 \%$, $37.17 \%$ and $56.4 \%$ respectively. Caused of much poor nutritional status in girls may be boys are given first priority with the available food within the family and the girls children were victim of negligence in the poor family due to lack of education. The nutrient intake of tribal children in study area it found that the diet system of their family were very poor. They were habituated to consume the rice and one locally available vegetable but not in recommended amount. Parent cannot buy a variety of vegetables. They are not able to talk milk, fat and oil and meat properly as a daily diet. Only one kind of food can use as daily food which are not sufficient for their body growth. The reason was their economically crisis and poor family background. So, the fewer in take in amount and the absence of variation in vegetable are causing in energy, vitamin and protein. All are directly related to malnutrition.

\section{References}

"World Bank Report", Source: The World Bank (2009). Retrieved 17.08.2011. "World Bank Report on malnutrition in India".

Adegun, J.A., Ajayi-Vinnet, O.B., and Alebiosu, E.O. Difference in the nutritional status of young school children from public and private owned primary schools in Ekiti State, Nigeria. European Scientific Journal. 2013, 9 (7). 
Amiral Ali El- Sabely, Hanan M.M.Tork and Yousria El- Sayid Hussien. Comparative study of nutritional status and Dietary habits of children from public and private primary Schools in Zagazig city, Egypt. IOSR Journal of Nursing and Health Science (IOSRJNHS). 2013, 3 (1): 47-52.

Amit Kaushik, Richa Raj, Chandra Patimishra, Srin Praksh Singh. Nutritional status of Rural Primary School Children and their SocioDemographic Correlates: A cross sectional study from Varanasi. Indian Journal of Community. 2012, 24:4.

Anand K, Kant S, Kapoo SK. Nutritional status of Adolescent school children in rural North India. Indian pediatric. 1999, 36 (8): 810-815.

Baliga S.S. Naik V.A, Mallapur M.D. Nutritional status of adolescent girls residing in rural area: A communitybased cross sectional study. Journal of the Scientific Society. 2004, 4 (1):2225.

Bhargava GK, Bhatt SC. Land and people of Indian states and Union Territories. 2006, 26, Tripura: Gyan Publishing House.

Caroline priya K. Seenivasan P, Praveen H, Amala Grace M, Annapoornai V, Shruthi Dhevi RS. A study on nutritional status of school children in rural, Semi-urban and urban areas of Tamil Nadu. Stanley Medical Journal. 2015, 2 (1): 3-11.

Chakraborty S., Bharti P. Nutritional status among the shabar tribal children living in urban, rural and forest habitats of Orissa, India. 2010, 7:3.

Chesire FJ, Organo AS, Oteba LP, Echoka E. Determinations of under-nutrition among school age children in a Nairobi peri-urban slum. East Afr Med. J. 2008, 85: 471- 479 .
Choudhury B. Social and Environmental dimensions of tribal health. In: S Basu (Ed): Tribal Health in India. Delhi: Manak publications Ltd., 1994, PP.7083.

Das H. $6^{\text {th }}$ "Left Front Government. Assumes office". People's democracy weekly organ of the communist party of India (Marxist); 2008, 32 (11).

Das S, Addhya D and Chakraborty F. Prevalence of thinness among 6-12 years rural children of Kharagpur, A cross sectional study in West Bengal, India. Anthrocon online Journal of Anthropology. 2012, 8: 5-10.

Das, Lipi, Bishnoi, Indira and Das, B.K. Anthropometric parameters for the assessment of nutritional status in (0-6) year's children in Varanasi. Man in India. 2000, 80 (3-4): 345-350.

Ew Mwaniki and A.N Mankokha. Nutritional status and associated factors among children in public primary school in Dagoretti, Nairobi, Kenya. African Health Sciences. 20`13; 13(1): 39-46.

Expert Group of the Indian Council of Medical Research, Nutrients Requirements and Recommended Dietary Allowances for Indians. New Delhi: ICMR. 1990.

Fazili A, Mir A, Pandit IM et al., Nutritional status of school age children (5-14 years) in a Rural Health Block of North India (Kashmir) using WHO z-score system. Online Journal of Health and allied Sciences. 2012; 11:1-3

Garba CMG, Mbofung CMF. Relationship between malnutrition and parasitic infection among school children in the Adamawa region of Cameroon. Pakistan Journal of Nutrition. 2010, 9: 1094-1099.

Global Monitoring Report 2012: Food prices nutrition and the millennium Development Goals. 
Gomez F, Galvan R, Frank S, Cravio to J, Chavez R, Vasquiz J. Mortality in second and third degree malnutrition. $\mathbf{J}$ Trop pediatric. 1956, 2: 77.

Gopal das T, Seshadri S. Nutrition Monitoring and assessment. New Delhi: Oxford University press. 1987.

Kalil S. and Z. Khan. A study of physical growth and Nutritional status of Rural School going children of Aligarh. Indian J. Prev. Soe. Med, 2004, 35: 3-4.

Lourie JA, Wiener JS. Assessment of nutritional status. In: JA Lourie, JS Wiener (Eds): Practical Human Biology. London: Susidiary of Harcourt Brace Javanovich publishers. 1981, pp. 27-52.

Panda P, Benjamin AL, Shavinder and Zachariah P. Health status of school children in Ludhiana city. Indian Journal of Community Medicine. 2000, 25 (4): 150-155.

Reji P, Belay G, Erko B, Mulugeta M, Belay M. Intestinal parasitic infections and malnutrition amongst first cycle primary school in Andama, Ethiopia. African Journal of Primary Health Care and Family Medicine 3. 2011.

Saheb sy, Rajendra Prasad M. Physical growth and nutritional status of the Lambadi children of Andra Pradesh. Anthropologist. 2009, 11: 195-206.
Satin V., Dhahliya. S. Nutritional assessment of rural school going children (7-9 years) of Hisar district, Haryana. Open Assess Scientific Report. 2002, 1(7): 24.

Srivastava A, Mahmood SE, Srivastava PM, Shrotriya VP, Kumar B. Nutritional status of school age children-A scenario of urban slums in India. Arc Public Health. 2012, 70: 8.

Sundaram MV, Sankaranarayanan VS, Susila R, Varalakshmi S, 1978. Health profile of school children in Madras city. Indian Pediatri, 15(9): 725-730.

Uddin MJ, Nag SK. Relationship of Socioeconomic status with anthropometric indicators of chronic energy deficiency among the rural adolescents of North Tripura, North East, India. Indian Journal of Physiology and Allied Science. 2012, 66 (2): 66-74.

WHO 1980. The Primary Health Worker. Geneva: World Health Organization.

WHO 1995. Physical status, the use and interpretation of Anthropometry. WHO Tec Rep Series No. 854, Geneva: WHO.

WHO 1999. Programming for adolescent Health and Development. WHO Tech Rep Series No. 886. Geneva: WHO.

\section{How to cite this article:}

Manabika Debbarma, Lord Litan Debbarma and Dipak Nath. 2018. Nutritional Health Status of Rural Tribal Children in Khowai District of Tripura. Int.J.Curr.Microbiol.App.Sci. 7(09): 31703183. doi: https://doi.org/10.20546/ijcmas.2018.709.395 